\title{
Axisymmetric equilibria with anisotropic resistivity and toroidal flow
}

\author{
G. Poulipoulis ${ }^{\dagger 1}$, G. N. Throumoulopoulos ${ }^{\dagger 2}$, H. Tasso ${ }^{\star 3}$ \\ ${ }^{\dagger}$ University of Ioannina, Association Euratom - Hellenic Republic, \\ Section of Theoretical Physics, GR 45110 Ioannina, Greece \\ *Max-Planck-Institut für Plasmaphysik, Euratom Association, \\ D-85748 Garching, Germany
}

\begin{abstract}
The equilibrium of an axisymmetric magnetically confined plasma with anisotropic resistivity and toroidal flow is investigated in the framework of magnetohydrodynamics (MHD). The stationary states are determined by an elliptic differential equation for the poloidal magnetic flux function $\psi$, a Bernoulli equation for the pressure and two relations for the resistivity components $\eta_{\|}$and $\eta_{\perp}$ parallel and perpendicular to the magnetic field. The flow can affect the equilibrium properties solely in the presence of toroidicity because in the limit of infinite aspect ratio the axial velocity does not appear in the equilibrium equations. The equilibrium characteristics of a tokamak with rectangular cross-section are studied by means of eigenfunctions in connection with exact solutions for the cases of "compressible" flows with constant temperature, $T(\psi)$, but varying density on magnetic surfaces and incompressible ones with constant density, $\varrho(\psi)$, but varying temperature thereon. Those eigenfunctions can describe either single or multiple toroidal configurations. In the former case the equilibrium has the following characteristics: (i) the $\eta_{\|^{-}}$ and $\eta_{\perp}$-profiles on the poloidal cross-section having a minimum close to the magnetic axis, taking large values on the boundary and satisfying the relation $\eta_{\perp}>\eta_{\|}$are roughly collisional (ii) the electric field perpendicular to the magnetic surfaces possesses two local extrema within the plasma and vanishes on the boundary and (iii) the toroidal current density is peaked close to the magnetic axis and vanishes on the boundary. The impact of the flow and the aspect ratio on the aforementioned quantities is evaluated for both "compressible" and incompressible flows.
\end{abstract}

\footnotetext{
${ }^{1}$ me00584@cc.uoi.gr

${ }^{2}$ gthroum@cc.uoi.gr

${ }^{3}$ het@ipp.mpg.de
} 


\section{Introduction}

Understanding the equilibrium properties of a magnetically confined plasma is one of the key issues in the fusion research. The majority of equilibrium studies up to date concern plasmas of zero electrical resistivity and have been based on the Grad-SchlüterShafranov equation which describes the magnetohydrodynamic (MHD) equilibria of axisymmetric plasmas. Additional physical input in terms of resistivity and plasma flow, however, are very important. In addition to its apparent role for Ohmic heating, the importance of resistivity is connected to the operation of a steady-state fusion reactor which will involve time scales much longer than the resistive-MHD one. Also an attractive feature of such a reactor would be operation under minimum sources of mass, momentum and energy. Sheared flow plays a key role in the formation of both edge transport barriers (L-H transition) $[1,2]$ and internal transport barriers $[3,4,5,6]$ in tokamaks. This flow is associated with sheared electric field profiles $[7,8,9,10]$ with significant amplitudes in the barrier region. Another pertinent quantity is the safety factor $[11,12,13]$ closely related to the toroidal current density.

Theoretically it was proved long time ago [14] that axisymmetric resistive MHD equilibria with scalar resistivity uniform on magnetic surfaces are not compatible with the Grad-Schlüter-Shafranov equation and the poloidal current density should vanish. The nonexistence of axisymmetric equilibria with constant resistivity was also suggested in Refs. [15, 16]. To examine whether these undesirable properties can be removed by including, in addition to resistivity in Ohm's law, flow and viscosity terms in the momentum equation is a formidable task, which should be preceeded by a step by step thorough investigation of particular cases possibly introducing additional physical input each time. In this respect two of the authors studied axisymmetric equilibria with scalar resistivity and flow purely toroidal [17] and parallel to the magnetic field $\mathbf{B}$ [18] and found that the flows considered can not remove the aforementioned inconsistencies. Non-vanishing poloidal currents are possible in steady states with parallel flows in the presence of anisotropy, i.e. for different resistivity components $\eta_{\|}$and $\eta_{\perp}$ parallel and perpendicular to $\mathbf{B}$ [19]; however, in this case neither $\eta_{\|}$nor $\eta_{\perp}$ can be uniform on magnetic surfaces. The sole external source in Refs. [17]-[19] is the toroidal current loop voltage. Resistive equilibria in a similar spirit of minimum external sources were investigated in Refs. $[15,16,20]$ and [21]-[23]. The particular flow directions considered in Refs. [17]-[19] are not inconsistent with Pfisrch-Schlüter diffusion in the sense that the equilibrium solutions constructed therein neither exclude nor can be included in possible Pfirsch-Schlüter-diffusion solutions (having velocity components perpendicular to the magnetic surfaces). Also, it is reminded that the pertinent pioneering study [24] does not include external sources of current and neglects the flow term in the momentum equation.

In this report we extend the studies [17]-[19] to equilibria with toroidal flows and anisotropic resistivity. There are two advantageous features of toroidal flows compared with parallel ones: (i) they are associated with electric fields $\mathbf{E}_{p}$ perpendicular to the magnetic surfaces and (ii) as we will show exact solutions with magnetic surfaces of uniform temperature, $T=T(\psi)$, are possible. Equilibria with incompressible flows having uniform density but varying temperature on magnetic surfaces will also be examined. In this respect it is noted that although purely toroidal axisymmetric flows are inherently incompressible because of symmetry, $T(\psi)$-equilibria can be regarded as "compressible" in the sense that the density varies on magnetic surfaces. In both cases the study can be 
carried out analytically up to the construction of exact solutions. In particular equilibrium eigenstates of a tokamak with rectangular cross-section will be derived in connection with exact solutions describing either single toroidal or multitoroidal configurations. Furthermore for single toroidal eigenstates we will study the characteristics of the conductivity components $\sigma_{\|}$and $\sigma_{\perp}$, the electric field $\mathbf{E}_{p}$, and the toroidal current density $J_{\phi}$ along with the impact of the flow on these quantities. This impact will be examined by varying a sound-speed Mach number $M_{0}$ for "compressible" flows and a parameter $A$ relating to the $\varrho$ - and $\mathbf{E}_{p^{-}}$profiles and their variation perpendicular to the magnetic surfaces for incompressible ones. In particular the uniformity of $\sigma_{\|}$and $\sigma_{\perp}$ on magnetic surfaces will be examined independently of solutions. Also it will be shown that the impact of the flow on the equilibrium is crucially related to the toroidicity.

An outline of the report is as follows. Reduced equilibrium equations involving $\psi$, the pressure and the resistivity components are derived in Sec. 2. Tokamak eigenstates are constructed in Sec. 3 for both "compressible" and incompressible flows. The equilibrium characteristics of $\sigma_{\perp}, \sigma_{\|}, \mathbf{E}_{p}$, and $J_{\phi}$ along with the impact of the flow and the aspect ratio on them is the subject of Sec. 4. The possible role of the flow shear is also briefly discussed therein. The conclusions are summarized in Sec. 5.

\section{Equilibrium equations}

In this section we shall derive reduced equilibrium equations for an axisymmetric magnetically confined plasma with anisotropic resistivity and toroidal flow. The procedure is unified in the sense that relevant energy equations or equations of state are not adopted from the beginning; they will specified when necessary later.

The starting equations in standard notation and convenient units are the following:

$$
\begin{array}{r}
\boldsymbol{\nabla} \cdot(\varrho \mathbf{v})=0, \\
\varrho(\mathbf{v} \cdot \boldsymbol{\nabla}) \mathbf{v}=\mathbf{J} \times \mathbf{B}-\nabla P, \\
\boldsymbol{\nabla} \times \mathbf{E}=0, \\
\boldsymbol{\nabla} \times \mathbf{B}=\mathbf{J}, \\
\boldsymbol{\nabla} \cdot \mathbf{B}=0, \\
\mathbf{E}+\mathbf{v} \times \mathbf{B}=\boldsymbol{\eta} \cdot \mathbf{J}=\eta_{\|} \cdot \mathbf{J}_{\|}+\eta_{\perp} \mathbf{J}_{\perp},
\end{array}
$$

where

$$
\boldsymbol{\eta}=\left(\begin{array}{cc}
\eta_{\|} & 0 \\
0 & \eta_{\perp}
\end{array}\right)
$$

is the resistivity tensor; the indices $\|$ and $\perp$ indicate directions parallel and perpendicular to $\mathbf{B}$; accordingly $\mathbf{J}_{\|}=(\mathbf{J} \cdot \mathbf{b}) \mathbf{b}, \mathbf{J}_{\perp}=\mathbf{b} \times(\mathbf{J} \times \mathbf{b})=\mathbf{J}-\mathbf{J}_{\|}$with $\mathbf{b}=\mathbf{B} / B$. The procedure to follow is based on identifying some integrals as flux functions, i.e. functions constant on magnetic surfaces, and reducing the set of Eqs. (1-6) by projecting the momentum equation (2) and Ohm's law (6) along the toroidal direction, the poloidal one (or parallel to B when convenient), and perpendicular to the magnetic surfaces. Important information is also drawn from an integral form of (6) [Eq. (13) below].

In cylindrical coordinates $(R, \phi, z)$ with $z$ corresponding to the axis of symmetry the equilibrium quantities for the case under consideration do not depend on the toroidal 
angle $\phi$; the toroidal velocity and the divergence-free magnetic field and current density can be expressed, with the aid of Ampére's law, in terms of the functions $K(R, z), \psi(R, z)$ and $I(R, z)$ as:

$$
\begin{array}{r}
\varrho \mathbf{v}=K \boldsymbol{\nabla} \phi \\
\mathbf{B}=I \nabla \phi+\nabla \phi \times \nabla \psi, \\
\mathbf{J}=\Delta^{*} \psi \nabla \phi-\nabla \phi \times \nabla I
\end{array}
$$

where $\psi$ labels the magnetic surfaces and $\Delta^{*} \equiv R^{2} \nabla \cdot\left(\nabla / R^{2}\right)$.

By projecting the momentum equation along the toroidal direction one obtains

$$
\nabla \phi \cdot(\nabla \psi \times \nabla I)=0
$$

which implies that $I=I(\psi)$. Therefor, unlike the case of parallel flow [19], the current surfaces coincide with the magnetic ones irrespective of equation of state. Integration of (6) along a contour $c$ defined by the cut of an arbitrary current surface with the poloidal plane yields the equation:

$$
\int_{c} \mathbf{E} \cdot d \mathbf{l}+\int_{c}(\mathbf{v} \times \mathbf{B}) \cdot d \mathbf{l}=\int_{c}(\boldsymbol{\eta} \cdot \mathbf{J}) \cdot d \mathbf{l},
$$

where $d \mathbf{l}=\boldsymbol{\nabla} \phi \times \nabla \psi /|\boldsymbol{\nabla} \phi \times \boldsymbol{\nabla} \psi|$ is the unit vector along the poloidal direction. Since in equilibrium it holds that $\partial \mathbf{B} / \partial t=0$, the first integral on the left-hand side of (13) vanishes by Stoke's theorem. Also the second integral vanishes due to the toroidal direction of the flow. For the integral on the right-hand side to vanish the integrand must necessarily do so because the $\mathbf{J}_{\text {pol }}$-lines are closed, nested and $\boldsymbol{\nabla} \cdot \mathbf{J}=0$; therefor it should hold locally

$$
(\boldsymbol{\eta} \cdot \mathbf{J}) \cdot d \mathbf{l}=(\boldsymbol{\eta} \cdot \mathbf{J})_{p o l}=0
$$

For isotropic resistivity, $\eta_{\perp}=\eta_{\|}$, (14) implies that the poloidal current density must vanish. In the presence of anisotropy, however, non-vanishing poloidal current densities are possible as expected because the toroidal electric field can drive a current in the poloidal direction. The rest of the report concerns equilibria with non-vanishing poloidal current densities.

Expressing the electric field on the poloidal cross-section in terms of the electrostatic potential, $\mathbf{E}_{p}=-\nabla \Phi$, the component of local Ohm's law (6) in the poloidal direction on account of (14) yields

$$
\boldsymbol{\nabla} \phi \cdot(\boldsymbol{\nabla} \Phi \times \boldsymbol{\nabla} \psi)=0 .
$$

which implies that $\Phi=\Phi(\psi)$; therefor $\mathbf{E}_{p}$ is perpendicular to the magnetic surfaces. The total electric field is given by

$$
\mathbf{E}=V_{c} \boldsymbol{\nabla} \phi+\mathbf{E}_{p}=V_{c} \boldsymbol{\nabla} \phi-\Phi^{\prime} \boldsymbol{\nabla} \psi
$$

where $2 \pi V_{c}$ is the constant toroidal loop voltage and the prime denotes derivative with respect to $\psi$. Subsequently, the component of (6) along $\nabla \psi$ yields

$$
\left(\Phi^{\prime}-\frac{K}{\varrho R^{2}}\right) \cdot|\nabla \psi|^{2}=0
$$


and therefor the quantity

$$
\frac{K}{\varrho R^{2}} \equiv \omega=\Phi^{\prime}
$$

identified as the rotation frequency, is a flux function $\omega=\omega(\psi)$. Eq. (14) and the component of (6) in the toroidal direction respectively yield the following equations:

$$
\begin{gathered}
-\frac{\Delta \eta}{(B R)^{2}}\left(I \Delta^{*} \psi-I^{\prime}|\nabla \psi|^{2}\right)-\eta_{\perp} I^{\prime}=0, \\
V_{c}=\Delta \eta \frac{I}{(B R)^{2}}\left[I^{\prime}|\nabla \psi|^{2}-I \Delta^{*} \psi\right]+\eta_{\perp} \Delta^{*} \psi,
\end{gathered}
$$

where $\Delta \eta=\eta_{\perp}-\eta_{\|}$. Any equilibrium solution should be compatible with (18) and (19) which, accordingly, can be solved for $\eta_{\perp}$ and $\eta_{\|}$to yield

$$
\begin{array}{r}
\eta_{\perp}=\frac{V_{c}}{\Delta^{*} \psi+I I^{\prime}}, \\
\eta_{\|}=\eta_{\perp}\left(1+\frac{I^{\prime}(B R)^{2}}{I \Delta^{*} \psi-I^{\prime}|\nabla \psi|^{2}}\right) .
\end{array}
$$

With the aid of the integrals $I=I(\psi), \Phi=\Phi(\psi)$ and $\omega=\omega(\psi)$ the components of (2) along $\mathbf{B}$ and $\nabla \psi$ respectively yield

$$
\begin{array}{r}
{\left[\frac{\nabla P}{\varrho}-\nabla\left(\frac{\omega^{2} R^{2}}{2}\right)\right] \cdot \mathbf{B}=0,} \\
{\left[\Delta^{*} \psi+I I^{\prime}\right]|\nabla \psi|^{2}+R^{2}\left[\nabla P-\varrho \omega^{2} \nabla\left(\frac{R^{2}}{2}\right)\right] \cdot \nabla \psi=0 .}
\end{array}
$$

Owing to the axisymmetry and the toroidal direction of the flow these equations do not contain the resistivity and are identical in form with the respective ideal-MHD equations.

In order to reduce Eqs. (22) and (23) further an energy equation or equation of state is necessary. Owing to the large heat conduction along $\mathbf{B}$, isothermal magnetic surfaces, $T=T(\psi)$, is an appropriate equation of state for fusion plasmas. In this case employing the ideal gas law, $P=\lambda \varrho T$, integration of (22) yields

$$
P=P_{s}(\psi) \exp \left(\frac{\omega^{2} R^{2}}{2 \lambda T}\right)
$$

where $P_{s}(\psi)$ is the pressure in the absence of flow. With the aid of (24), Eq. (23) leads to the final "compressible" equation

$$
\Delta^{*} \psi+I I^{\prime}+R^{2}\left[P_{s}^{\prime}+P_{s} \frac{R^{2}}{2}\left(\frac{\omega^{2}}{\lambda T}\right)^{\prime}\right] \exp \left(\frac{\omega^{2} R^{2}}{2 \lambda T}\right)=0 .
$$

For ideal plasmas $(\boldsymbol{\eta}=\mathbf{0})$ Eq. (25) was originally obtained in Ref. [25].

An alternative equation of state is incompressibility:

$$
\boldsymbol{\nabla} \cdot \mathbf{v}=0
$$

Consequently, (1) implies that the density is a flux function, $\varrho=\varrho(\psi)$, and therefor one can find along the same lines the following incompressible equations for $P$ and $\psi$ :

$$
P=P_{s}(\psi)+\frac{R^{2} \varrho \omega^{2}}{2}
$$




$$
\Delta^{*} \psi+I I^{\prime}+R^{2} P_{s}^{\prime}+\frac{R^{4}}{2}\left(\varrho \omega^{2}\right)^{\prime}=0 .
$$

Eq. (28) is identical with a particular form of the axisymmetric equilibrium equation for incompressible flow of arbitrary direction obtained in Ref. [26] for ideal plasmas.

Once Eqs. (25) and (28) are solved for $\psi$ the resistivity components can be determined by (20) and (21). In general inspection of Eqs. (20) and (21) implies, like the case of parallel flows [19], that neither $\eta_{\perp}$ nor $\eta_{\|}$can be uniform on magnetic surfaces; indeed solving (25) and (28) for $\Delta^{\star} \psi$ and substituting into (20) and (21), one can see that $\eta_{\perp}$ and $\eta_{\|}$depend, in addition to $\psi$, explicitly on $R$ (and on $|\nabla \psi|^{2}$ as concerns $\left.\eta_{\|}\right)$. However as we will see in Sec. $4, \eta_{\perp}$ and $\eta_{\|}$can be collisional-like, viz. they can have a minimum close to the magnetic axis, take very large values on the boundary and it holds that $\eta_{\perp}>\eta_{\|}$. Reasons for temperature deviations on magnetic surfaces, which can result to non uniformity of $\eta_{\|}$and $\eta_{\perp}$ thereon, are discussed in Sec. III of Ref. [19].

Summarizing this section, the MHD equilibrium states of an axisymmetric plasma with anisotropic resistivity and toroidal flow is governed by an elliptic differential equation for the poloidal magnetic flux function [Eq. (25) for "compressible" flow and (28) for incompressible one], a Bernoulli relation for the pressure and self-consistent expressions for the resistivities $\eta_{\|}$and $\eta_{\perp}$. Both Eqs. (25) and (28) contain four flux-functions, three out of which, i.e. $P_{s}, I$ and $\omega$, are common. The fourth function is $T$ for the "compressible equation" and $\varrho$ for the incompressible one. For vanishing flow (25) and (28) reduce to the Grad-Schlüter-Shafranov equation.

\section{Exact solutions}

Linearized forms of Eqs. (25) and (28) in connection with appropriate assignments of the free flux functions they contain can be solved analytically. In the present study we will employ exact solutions as follows.

"Compressible" flow

The ansatz used to linearize Eq. (25) is [27] [28]

$$
\begin{array}{r}
I^{2}=I_{0}^{2}+I_{1}^{2} \psi^{2} \\
P_{s}=2 P_{0} \psi^{2} \\
\frac{\omega^{2}}{\lambda T}=\frac{\gamma M_{0}^{2}}{R_{0}^{2}}=\text { constant }
\end{array}
$$

Here, $I_{0} / R$ is the toroidal vacuum field, the parameter $I_{1}$ describes the magnetic properties of the plasma; $P_{0}, \gamma$, and $M_{0}$ are a pressure parameter, the ratio of specific heats, and the Mach number with respect to the sound-speed at a reference point $\left(z=0, R=R_{0}\right)$ with $R_{0}$ to be specified later. Note that the toroidal current density profile can vanish on the plasma boundary via (10).

Eq. (25) then has a separable solution, $\mathcal{R}(R) \mathcal{Z}(z)$, when the constant of separation is equal to $R_{0} I_{1}$. For configurations symmetric with respect to mid-plane $z=0$ this solution is written in the form

$$
\psi(x, y)=C_{1}\left[J_{0}\left(\frac{2 \tau \sqrt{e^{\frac{\gamma M_{0}^{2} x^{2}}{2}}}}{\gamma M_{0}^{2}}\right)+C_{2} Y_{0}\left(\frac{2 \tau \sqrt{e^{\frac{\gamma M_{0}^{2} x^{2}}{2}}}}{\gamma M_{0}^{2}}\right)\right] \cos \left(R_{0} I_{1} y\right)
$$


where $x=R / R_{0}$ and $y=z / R_{0} ; J_{0}$ and $Y_{0}$ are zeroth-order Bessel functions of first- and second-kind respectively; and $\tau^{2} \equiv 4 P_{0} R_{0}^{4}$.

Incompressible flow

In this case the ansatz employed to linearize (28) is [29]

$$
\begin{array}{r}
I^{2}=I_{0}^{2}+I_{1}^{2} \psi^{2} \\
P_{s}=2 P_{0} \psi^{2} \\
\left(\varrho \omega^{2}\right)^{\prime}=\left[\frac{K^{2}}{\varrho R^{4}}\right]^{\prime}=2 A \psi
\end{array}
$$

The third of equations (31) on account of (17) indicates that $A$ is associated with the density and electric field profiles and their variation (shear) perpendicular to the magnetic surfaces $\left[\left(\varrho \omega^{2}\right)^{\prime} \neq 0\right]$. The polarity of $\mathbf{E}_{p}$ and the aforementioned shear permits $A$ to take either positive or negative values. This is a remarkable difference as compared with the choice (29) which is shearless $\left[\left(\omega^{2} / \lambda T\right)^{\prime}=0\right]$. Also, note that, unlike $M_{0}$ in (29), $A$ is dimensional.

A separable solution is now expressed in terms of the first- and second-kind Airy functions, $A_{i}$ and $B_{i}$, as [29]

$$
\begin{array}{r}
\psi(x, y)=C_{1}\left[A i\left(\left(\frac{A R_{0}}{4}\right)^{-2 / 3}\left(\frac{A R_{0}^{6}}{4} x^{2}-P_{1} R_{0}^{4}\right)\right)\right. \\
\left.+C_{2} B i\left(\left(\frac{A R_{0}}{4}\right)^{-2 / 3}\left(\frac{A R_{0}^{6}}{4} x^{2}-P_{1} R_{0}^{4}\right)\right)\right] \cos \left(R_{0} I_{1} y\right) .
\end{array}
$$

In connection with solutions (30) and (32) we are interested in the steady states of a tokamak the plasma of which is bounded by a conducting wall of rectangular cross-section, as shown in Fig. 1. In addition, we assume that the plasma boundary coincides with the outermost magnetic surface. Thus, the magnetic field is tangential to and the pressure $P$ must vanish on the boundary [30]; accordingly, the function $\psi$ should satisfy the following boundary conditions

$$
\psi\left(y_{ \pm}\right)=0
$$

and

$$
\psi\left(x_{ \pm}\right)=0
$$

where $y_{ \pm}= \pm a / R_{0}$ and $x_{ \pm}=1 \pm b / R_{0}$. The equilibrium becomes then a boundary-value problem. Eigenstates can be determined by imposing conditions (33) and (34) directly to solutions (30) and (32). Specifically, (33) applied to the $z$-dependent part of the solutions yields the eigenvalues

$$
I_{1}^{\ell}=\frac{1}{\mathrm{a}}\left(\ell \pi-\frac{\pi}{2}\right), \quad \ell=1,2, \ldots
$$

for the quantity $I_{1}$ which is related to the poloidal current function $I(\psi)$. The respective eigenfunctions are associated with configurations possessing $\ell$ magnetic axes parallel to the axis of symmetry. Condition (34) is pertinent to the $R$-dependent part of the solution. Owing to the flow this part contains the parameter $M_{0}$ in the "compressible" case and $A$ in the incompressible one in addition to the pressure parameter $P_{0}$. Thus, condition (34) can determine flow eigenvalues depending on the parameter $P_{0}$ which remains free, 
$F^{n}\left(P_{0}\right)(n=1,2,3, \ldots)$ with $\mathrm{F}$ standing for either $M_{0}$ or $A$, or vice versa, pressure eigenvalues $P_{0}^{n}(F)$ with $F$ being free. The other parameters $C_{1}$ and $C_{2}$ contained in (30) and (32) are adapted to normalize $\psi$ with respect to the magnetic axis and to satisfy the boundary condition (34) respectively. The eigenfunctions in association with $F^{n}\left(P_{0}\right)$ [or $\left.P_{0}^{n}(F)\right]$ are connected to configurations having $n$ magnetic axes perpendicular to the axis of symmetry. Therefor the total equilibrium eigenfunctions $\psi_{\ell n}=\mathcal{Z}_{\ell}(z) \mathcal{R}_{n}(R)$ describe multitoroidal configurations having $\ell \times n$ magnetic axes.

On the basis of the above solutions one can evaluate the impact of the flow on the resistivity components $\eta_{\perp}$ [Eq. (20)] and $\eta_{\|}$[Eq. (21)], the electric field perpendicular to the magnetic surfaces $\left[\mathbf{E}_{p}=-\nabla \Phi=-\Phi^{\prime} \boldsymbol{\nabla} \psi\right]$ and the toroidal current density $J_{\phi}$ [Eq. (11)] for both "compressible" and incompressible flows. It is emphasized that this impact is crucially related to the toroidicity because in the limit of infinite aspect ratio the equilibrium equations do not contain the axial velocity regardless of "compressibility". Indeed, for a cylindrical plasma of arbitrary cross-section the equations respective to (22) and (23) read

$$
\begin{aligned}
\text { B } \cdot \nabla P & =0 \\
\nabla^{2} \psi+\left(P+\frac{B_{z}^{2}}{2}\right)^{\prime} & =0 .
\end{aligned}
$$

For ideal plasmas Eqs. (36) and (37) follow respectively from (16) and (17) of Ref [31] for vanishing poloidal velocity $\left(F^{\prime}=0\right.$ therein). Therefor the flow may have an impact on equilibrium only in the presence of toroidicity. Also, note that the pressure becomes a flux-function. Because of the importance of toroidicity the impact of the aspect ratio on equilibrium in addition to that of the flow will be evaluated in the next section.

\section{Impact of the flow and aspect ratio on equilibrium}

The quantities to be examined are the conductivity components $\sigma_{\perp}=1 / \eta_{\perp}$ and $\sigma_{\|}=$ $1 / \eta_{\|}$, the electric field $\mathbf{E}_{p}$ and the toroidal current density $J_{\phi}$. Both "compressible" and incompressible flows will be studied for single toroidal configurations. The study is based on the eigenfunction $\psi_{11}$ which for "compressible" flow is shown in Fig. 2 and will be made by varying the flow parameters $M_{0}$ and $A$ for "compressible" and incompressible flows respectively. For any value of $M_{0}$ or $A$ the respective lowest eigenvalue of the pressure parameter $P_{0}$ will be calculated numerically. The variation of $M_{0}$ and $A$ will correspond to the same range of eigenvalues of $P_{0}$. Specifically, for aspect ratio $\alpha=3$ the flow parameters $M_{0}$ and $A$ will be ranged in the intervals $[0.1,0.7]$ and $[-0.001,-0.01]$ respectively. For $\alpha=2$ the respective intervals will be $[0.1,1]$ and $[-0.001,-0.08]$ unless stated otherwise. Variation of the flow parameters in connection with the results to be presented will refer to these intervals. The tokamak scaling $B_{p} \approx 0.1 B_{\phi}$ will be used for the calculations. Also, we should make the following clarification: it occurs that solutions (30) and (32) oscillate as the flow parameters are varied, viz. for any given point $(R, z)$ these solutions considered as functions $\psi\left(M_{0}\right)$ and $\psi(A)$ take successively larger and lower values as $M_{0}$ and $A$ are varied monotonically. This, would give rise to an oscillatory behavior to all physical quantities which is physically unjustifiable. For the conductivity components this can be seen in Fig. 3. To avoid this difficulty the solutions will be normalized in such a way that the poloidal magnetic flux on the magnetic axis is unity irrespective of 
flow. This is accomplished by choosing appropriately the parameter $C_{1}$ for each value of $M_{0}$ or $A\left[C_{1}\left(M_{0}\right)\right.$ for "compressible" flow and $C_{1}(A)$ for "incompressible" one]. Also, solutions (30) and (32) have a strong parametric dependence on the flow parameters [note the exponential dependence of (30) on $M_{0}^{2}$ ]. Consequently, this dependence results in large quantitative changes in the physical quantities for large $M_{0}\left(M_{0} \approx 1\right)$ or small $A$ ( $A \approx-0.01)$ which most probably overestimate the impact of flow. In addition, it is noted that except for the conductivity components an increase of $M_{0}$ has qualitatively the same impact on the physical quantities to be examined with that caused by a decrease of A.

The results concerning the characteristics of the quantities $\sigma_{\perp}, \sigma_{\|}, \mathbf{E}_{p}$, and $J_{\phi}$ and the impact of the flow and $\alpha$ on them are as follows.

\section{Conductivity components}

1. For both "compressible" and incompressible flows the profiles of $\sigma_{\perp}$ and $\sigma_{\|}$on the poloidal cross-section are collisional-like, i.e. they have a maximum close to the magnetic axis vanish on the boundary and stands in most of the cases that $\sigma_{\|}>$ $\sigma_{\perp}[33]$. Profiles of the resistivity components on the mid-plane $z=0$ are shown in Fig. 4 for "compressible" flows and in Fig. 5 for incompressible ones. For vanishing flow and $\alpha=3$ one finds $\Delta \sigma / \sigma_{\|} \equiv\left(\sigma_{\|}-\sigma_{\perp}\right) / \sigma_{\|}=0.2122$; for the reversed-fieldpinch scaling, $B_{\phi} \approx B_{p}$, the value of $\left(\sigma_{\|}-\sigma_{\perp}\right) / \sigma_{\|}$becomes double, i.e. 0.4244. Also, for $\alpha=3$ increase of $M_{0}$ from 0.1 to 0.7 results in a percentage decrease of $\Delta \sigma$ by $4 \%$ while decrease of $A$ from -0.001 to -0.01 leads to an increase of $\Delta \sigma$ by $3.4 \%$.

2. The maximum of the profiles takes larger values as $M_{0}$ increases and lower values as $A$ decreases. For $\alpha=3$ increase of $M_{0}$ (from 0.1 to 0.7 ) leads to percentage increases of $\sigma_{\perp}$ and $\sigma_{\|}$by $9 \%$ and $7 \%$ respectively. For incompressible flows the respective decreases are $73 \%$ and $67 \%$ (for $\alpha=3$ and decrease of $A$ from -0.001 to -0.01).

3. The position of the maximum is shifted outwards from the axis of symmetry as $M_{0}$ increases and is nearly insensitive to the variation of $A$ [32] (see Figs. 4 and 5). For example for $\alpha=3$ the position of the maximum of $\sigma_{\perp}$ changes from 1.119 to 1.151 (when $M_{0}$ varies from 0.1 to 0.7 ).

4. The lower $\alpha$

- the larger the decrease of $\Delta \sigma=\sigma_{\|}-\sigma_{\perp}$ as $M_{0}$ increases;

- the smaller the increase of $\Delta \sigma$ as $A$ decreases;

- the larger the increase of the $\sigma_{\perp-}$ and $\sigma_{\|}$-maximum as $M_{0}$ increases;

- the smaller the decrease of the $\sigma_{\perp}$ - and $\sigma_{\|}$-maximum as $A$ decreases;

- the larger the shift of the position of $\sigma_{\perp}$ - and $\sigma_{\|}$-maximum as $M_{0}$ increases.

Those conclusions become evident if the following results for $\alpha=2$ are compared with those presented above for $\alpha=3$ :

- the decrease of $\Delta \sigma$ for compressible flow is $13 \%$;

- the increase of $\Delta \sigma$ for incompressible flow is $1 \%$. The respective variation of $A$ in this case is $[-0.001,-0.01]$ (see [33]); 
- the increase of the conductivity maximum values for "compressible" flows are $44 \%\left(\sigma_{\perp}\right)$ and $27 \%\left(\sigma_{\|}\right)$;

- the decrease of the conductivity maximum values for incompressible flows are $43.7 \%\left(\sigma_{\perp}\right)$ and $63 \%\left(\sigma_{\|}\right)$;

- the position of the maximum of $\sigma_{\perp}$ for "compressible" flow is displaced from 1.228 to 1.33 .

The "compressibility" dependent impact of the flow on $\sigma_{\perp}, \sigma_{\|}$and $\Delta \sigma$ may relate to the flow shear of the incompressible solution (32) while the "compressible" solution (30) is shearless.

\section{Electric field}

Prescribing the rotation frequency $\omega(\psi)$ by

$$
\omega=\omega_{0} \psi^{n}
$$

where $n$ is a shaping parameter, the electric field, $\mathbf{E}_{p}=-\Phi^{\prime} \nabla \psi$, with the help of (17) becomes

$$
\mathbf{E}_{p}=-\omega_{0} \psi^{n} \nabla \psi
$$

The profile of $\mathbf{E}_{p}$ on the poloidal cross-section possesses two local extrema within the plasma volume one on the left-hand-side and the other on the right-hand-side of the magnetic axis with opposite sign, which means that the electric filed has different polarity [as can also be deduced from Eq. (39)] and is similar to ones observed in equilibria with internal transport barriers. The profile $\left|E_{r}\right|$, on the mid-plane $y=0$ is shown in Fig. 6 . Increase of $M_{0}$ or decrease of $A$ leads to an increase of both local maximum values of $\left|E_{r}\right|$ with the one outwards more than the one inwards the magnetic axis and to a displacement of their positions outwards. For "compressible" flows this is shown in (Fig. 6). For $\alpha=3$ the maximum values of $\left|E_{r}\right|$ increase by $4 \%$ for "compressible" flow (increase of $M_{0}$ from 0.1 to 0.7 ) and $5.6 \%$ for incompressible one (decrease of $A$ from -0.001 to -0.01 ). The lower $\alpha$ the larger the increase of the maxima and the displacement of their positions outwards. As an example, for $\alpha=2$ the increase of the maximum $\left|E_{r}\right|$ becomes as large as $15 \%$ for "compressible" flow with strong increase occurring above $M_{0}=0.8$ and $9 \%$ for incompressible flow. Also, when the parameter $n$ takes larger values the maxima of $\left|E_{r}\right|$ take lower values but the profile of $E_{r}$ becomes steeper and more localized (Fig. 7); therefor the shear $S_{E_{r}}=\partial E_{r} / \partial x$ is also increased.

\section{The toroidal current density}

The profile of the toroidal current density,

$$
J_{\phi}=\frac{1}{R} \Delta^{*} \psi
$$

is peaked with its maximum in the vicinity of the magnetic axis and vanishes on the plasma boundary (Fig. 8). Like $\mathbf{E}_{p}$, as $M_{0}$ increases or $A$ decreases the maximum of $J_{\phi}$ takes larger values and its position is displaced outwards. Also, the impact of the variation of the flow parameters is stronger as the aspect ratio becomes smaller. In particular for $\alpha=3$ and $\alpha=2$ the maximum value of the current density increases by $8 \%$ and 
$35.5 \%$ respectively for compressible flows (increase of $M_{0}$ ). The respective increases for incompressible flows (decrease of $A$ ) are $5.6 \%$ and $18 \%$.

\section{Conclusions}

In this report we have investigated the MHD equilibrium of an axisymmetric magnetically confined plasma with anisotropic resistivity and toroidal flow subjected to the single external source of toroidal current loop voltage by including the flow term in the momentum equation. Equilibria of this kind are inherently free of Pfirsch-Schlüter diffusion. Also, unlike the case of parallel flows, there is an electric field $\mathbf{E}_{p}$ perpendicular to the magnetic surfaces. The study includes "compressible" flows with varying density but uniform temperature on magnetic surfaces and incompressible ones with uniform density but varying temperature thereon. It turns out that the equilibrium states are determined by an elliptic differential equation for the poloidal magnetic flux function $\psi$ [Eq. (25) for compressible flows and (28) for incompressible one], a Bernoulli equation for the pressure [respective Eqs. (24) and (27)] and two relations for the resistivities $\eta_{\perp}$ [Eq. (20)] and $\eta_{\|}$[Eq. (21)] parallel and perpendicular to the magnetic field. Owing to axisymmetry and the toroidal direction of the flow, the equilibrium equations and pressure relations are identical in form with the respective ideal-MHD ones. The impact of the flow on equilibrium can be "activated" solely in the presence of toroidicity because the cylindrical equilibrium equations do not contain the axial velocity.

The equilibrium of a tokamak plasma bounded by a rectangular cross-section has been studied by means of equilibrium eigenfunctions in connection with exact solutions for "compressible" and incompressible flows. These eigenfunctions can describe either single toroidal or multiple toroidal configurations. In the former case we have studied the characteristics of the conductivities $\sigma_{\perp}$ and $\sigma_{\|}$, the electric field $\mathbf{E}_{p}$, and the toroidal current density $J_{\phi}$ as well as how they are affected by the flow. The impact of the flow has been examined by varying pertinent flow parameters, i.e. a sound-speed Mach number $M_{0}$ for the "compressible" flow and a parameter $A$ relating to the density and electric field and their variations perpendicular to the magnetic surfaces for the incompressible one. For (i) single toroidal configurations (ii) eigenfunctions normalized so that $\psi$ is unity on the magnetic axis regardless of flow (a normalization made to avoid physically unjustifiable oscillation of the solutions on the flow parameters) and (iii) variation of the flow parameters corresponding to the same variation of the lowest eigenvalue for the pressure parameter $P_{0}$ we came to the following conclusions:

1. For a toroidal frequency $\omega(\psi)$ peaked on the magnetic axis and vanishing on the boundary [Eq. (38)] the profile of $\left|\mathbf{E}_{p}\right|$ on the poloidal cross-section has two maxima located the one on the left-hand-side and the other on the right-hand-side of the magnetic axis and it vanishes on the boundary. When the maximum of $\omega$ becomes larger and its profile more localized, the profile of $\left|\mathbf{E}_{p}\right|$ becomes as well more localized though its maxima become smaller.

2. The profile of $J_{\phi}$ is peaked with its maximum close to the magnetic axis and vanishes on the boundary.

3. Although the conductivity components can not be uniform on magnetic surfaces (this follows in general by inspection of the equilibrium equations) their profiles are 
roughly collisional, viz. they have a maximum in the vicinity of the magnetic axis, vanish on the boundary and it holds, in most of the cases that $\sigma_{\|}>\sigma_{\perp}$.

4. As $M_{0}$ increases or $A$ decreases the local maxima of $\mathbf{E}_{p}$ and $J_{\phi}$ take larger values and their positions are shifted outwards from the axis of symmetry.

5. The impact of the variation of the flow parameters on $\sigma_{\perp}$ and $\sigma_{\|}$rely on "compressibility": like $\mathbf{E}_{p}$ and $J_{\phi}$, the maxima of $\sigma_{\perp}$ and $\sigma_{\|}$become larger as $M_{0}$ increases and their positions are displaced outwards but these maxima become smaller and their positions are nearly not affected as $A$ decreases. Also, the larger the $M_{0}$ the smaller $\sigma_{\|}-\sigma_{\perp}$ but the smaller $A$ the larger $\sigma_{\|}-\sigma_{\perp}$.

6. For a given value of $M_{0}$, the lower the aspect ratio $\alpha$ the smaller the maxima of $\sigma_{\|}$, $\sigma_{\perp}$ and $J_{\phi}$ but the larger the maximum of $\mathbf{E}_{p}$.

7. For a given value of $A$, the lower the aspect ratio $\alpha$ the larger the maxima of $\sigma_{\|}, \sigma_{\perp}$ and $\mathbf{E}_{p}$ but the smaller the maximum of $J_{\phi}$.

8. For increase of $M_{0}$ or decrease of $A$ (corresponding to the same variation of the lowest eigenvalue of $P_{0}$ ), the lower $\alpha$ the higher the variation of the maxima of $\mathbf{E}_{p}$ and $J_{\phi}$ and the displacements of their positions outwards. The impact of $\alpha$ on the conductivities are "compressibility" dependent: the smaller the $\alpha$ (i) the larger the variation of the maximum of $\sigma_{\perp}, \sigma_{\|}$and $\sigma_{\|}-\sigma_{\perp}$ (when $M_{0}$ increases) but (ii) the smaller the maximum of $\sigma_{\perp}, \sigma_{\|}$and $\sigma_{\|}-\sigma_{\perp}$ (when $A$ decreases).

Qualitatively, except for the conductivity components the impact of the flow on $\mathbf{E}_{p}$ and $J_{\phi}$ are independent of "compressibility". The dependence of the results for $\sigma_{\perp}$ and $\sigma_{\|}$ on "compressibility" may be due to the fact that the the incompressible solution (32) has finite flow shear while the flow of the compressible one (30) is shearless. Quantitatively, for $\alpha=2$ and increase of $M_{0}$ from 0.1 to 0.5 or decrease of $A$ from -0.001 to -0.006 result in percentage variations of all quantities $\left(\sigma_{\|}, \sigma_{\perp}, \mathbf{E}_{p}\right.$, and $\left.J_{\phi}\right)$ less that $10 \%$. Larger variations of these quantities associated with higher values of $M_{0}$ or lower values of $A$, we have found on the basis of solutions (30) and (32), most probably overestimate the actual impact of the flow.

\section{Acknowledgement}

Part of this work was conducted during a visit of the authors G.P. and G.N.T. to the MaxPlanck-Institut für Plasmaphysik, Garching. The hospitality of that Institute is greatly appreciated. 


\section{References}

[1] P. W. Terry, Rev. Mod. Phys. 72(2000), 109.

[2] J.S. deGrassie et al, Phys. Plasmas 11(2004) 4323.

[3] A.K. Wang et al, Phys. Plasmas 9(2002) 748.

[4] C.L. Fiore et al, Phys. Plasmas 8(2001) 2023.

[5] J.E. Rice et al, Nucl. Fusion 41(2001) 277.

[6] J.W. Connor et al, Nucl. Fusion 44 (2001) R1.

[7] K.H. Burell et al, Phys. Plasmas 1(1994) 1536.

[8] E. Mazzucato et al, Phys. Rev. Lett. 77(1996) 3145.

[9] R.E. Bell et al, Phys. Rev. Letters 81(1998) 1429.

[10] H. Meister et al, Nucl. Fusion 41(2001) 1633.

[11] E. Joffrin et al, Plasma Phys. Control. Fusion 44(2002) 1739.

[12] X. Litaudon et al, Plasma Phys. Control. Fusion 38(1996) 1603.

[13] E.D. Quigley et al, Nucl. Fusion 44(2004) 1189.

[14] H. Tasso, Lectures on Plasma Physics, Report IFUSP/P-181, LFP-8, Universidade de São Paulo, Instituto de Física, São Paulo (1979), p. 27. See EPAPS Document No. EPHPAEN-10-027306 for Lectures on Plasma Physics. A direct link to this document may be found in the online article's HTML reference section. The document may also be reached via the EPAPS homepage (http://www.aip.org/pubservs/epaps.html) or from ftp.aip.org in the directory /epaps/. See the EPAPS homepage for more information.

[15] D. Montgomery, and X. Shan, Comments Plasma Phys. Controlled Fusion 15 (1994), 315.

[16] D. Montgomery, J.W. Bates, and H.R. Lewis, Phys. Plasmas 4 (1997), 1080.

[17] G.N. Throumoulopoulos, J. Plasma Physics 59 (1998), 303

[18] G.N. Throumoulopoulos and H. Tasso, J. Plasma Physics 64 (2000), 601.

[19] G.N. Throumoulopoulos and H. Tasso, Phys. Plasmas 10, 2382 (2003).

[20] L.P. Kamp and D. Montgomery, Phys. Plasmas 10 (2003), 157.

[21] J.W. Bates and H.R. Lewis, Phys. Plasmas 3 (1996), 2395.

[22] M.L. Goodman, J. Plasma Physics 60 (1998), 587.

[23] M.P. Bora, Phys. Plasmas 7 (2000), 3097. 
[24] D. Pfirsch and A. Schlüter, Der Einfluß der elektrischen Leitfähigkeit auf das Gleichgewichtsverhalten von Plasmen niedringen Drucks in Stellaratoren, Report MPI/PA/7/62, 1962, Max-Planck-Institut, Munich; available also as National Technical Information Document No. MPI/PA/7/62, Munich, 1962 by D. Pfirsch and A. Schlüter. Copies may be ordered from the National Technical Information Service, Springfield, VA 22161; See also J. Wesson, Tokamaks 2nd ed. [Oxford Science, New York (The Oxford engineering science series), 1997], p. 48.

[25] E.K. Maschke and H. Perrin, Plasma Phys. 22 (1980), 579.

[26] H. Tasso and G.N. Throumoulopoulos, Phys. Plasmas 5 (1998), 2378.

[27] R.A. Clemente and R. Farengo, Phys. Fluids 27 (1984), 776.

[28] G.N. Throumoulopoulos and G. Pantis, Phys. Fluids B 1 (1989), 1827.

[29] G.N. Throumoulopoulos, G. Poulipoulis, G. Pantis, H. Tasso, Phys. Lett. A $\mathbf{3 1 7}(2003), 463$.

[30] The consequences of the toroidal electric field on the boundary conditions are discussed in more detail in Refs. [17] and [18].

[31] G.N. Throumoulopoulos and H. Tasso, Phys. Plasmas 4 (1997), 1492.

[32] To be precise, for incompressible flow when $A$ decreases the position of the maximum first is shifted outwards and then inwards when $A$ take small negative values.

[33] For $\alpha=2$ and very small algebraic values of $A$ i.e. $A \leq-0.08$ it holds that $\sigma_{\|}<\sigma_{\perp}$. 


\section{Figure captions}

Fig. 1: The cross-section of the plasma boundary. The aspect ratio $\alpha$ is defined as $R_{0} / b$, where $R_{0}$ is the geometric center of the configuration.

Fig. 2: Magnetic surface cross-sections for the single toroidal configuration in connection with the eigenfunction $\psi_{11}$ for $M_{0}=0.5$ and $\alpha=3$.

Fig. 3: A set of curves demonstrating the oscillation of the profile of $\sigma_{\perp}$ on the mid-plane $z=0$, normalized with respect to a constant value $\sigma_{c}$, for "compressible" flow when the value of the function $\psi$ on the magnetic axis is flow dependent and the Mach number $M_{0}$ increases: a) $M_{0}=0.1$, b) $M_{0}=0.2$, c) $M_{0}=0.5$ and d) $M_{0}=0.6$. The aspect ratio is $\alpha=2 ; x \equiv R / R_{0}$ with the vertical axis being placed at the position of the geometric center $(x=1)$.

Fig. 4: The figure shows the increase of the maximum of $\sigma_{\perp}$ and $\sigma_{\|}$and the displacement of its position outwards for "compressible" flow due to the increase of the Mach-number $M_{0}$.

Fig. 5: The figure shows the decrease of the maximum of $\sigma_{\perp}$ and $\sigma_{\|}$but the insensitivity of its position for incompressible flow as the parameter $A$ decreases.

Fig. 6: Electric field profiles on the mid-plane $z=0$ for "compressible" flow Comparison of the two curves indicates the increase of the local maxima of $\mathbf{E}_{p}$ and the outward shift of the their positions as the Mach number $M_{0}$ increases from 0.8 to 1 . The point in between the two maxima at which $\mathbf{E}_{p}=0$ corresponds to the magnetic axis. The aspect ratio is $\alpha=2$ and the value of the shaping parameter $n$ [Eq. (38)] is 3 .

Fig. 7: Electric field profiles on the mid-plane $z=0$ for $\alpha=3, M_{0}=0.4$ and two different values of the shaping parameter $n: n=1$ and $n=3$.

Fig. 8: Toroidal current density profiles on the mid-plane $z=0$ for "compressible" flows Comparison of the two curves indicates the increase of the maximum and the outward displacement of its position as the Mach number $M_{0}$ increases from 0.1 to 0.8 . The aspect ratio is $\alpha=2$. 


\section{List of Figures}

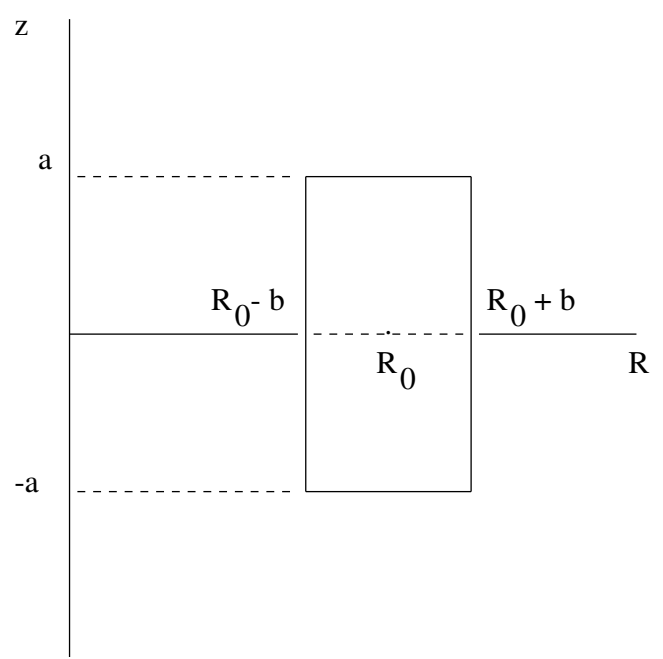

Figure 1: The cross-section of the plasma boundary. The aspect ratio $\alpha$ is defined as $R_{0} / b$, where $R_{0}$ is the geometric center of the configuration.

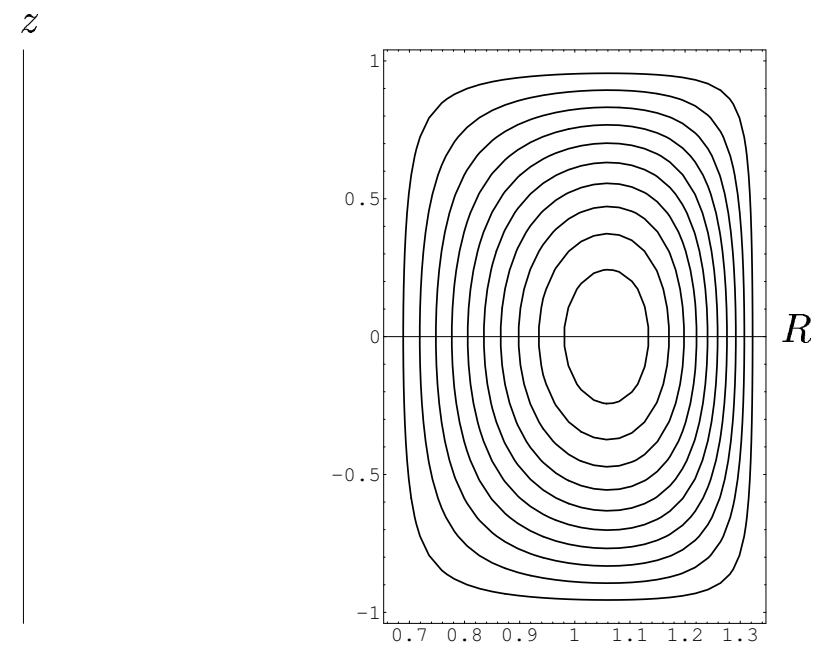

Figure 2: Magnetic surface cross-sections for the single toroidal configuration in connection with the eigenfunction $\psi_{11}$ for $M_{0}=0.5$ and $\alpha=3$. 

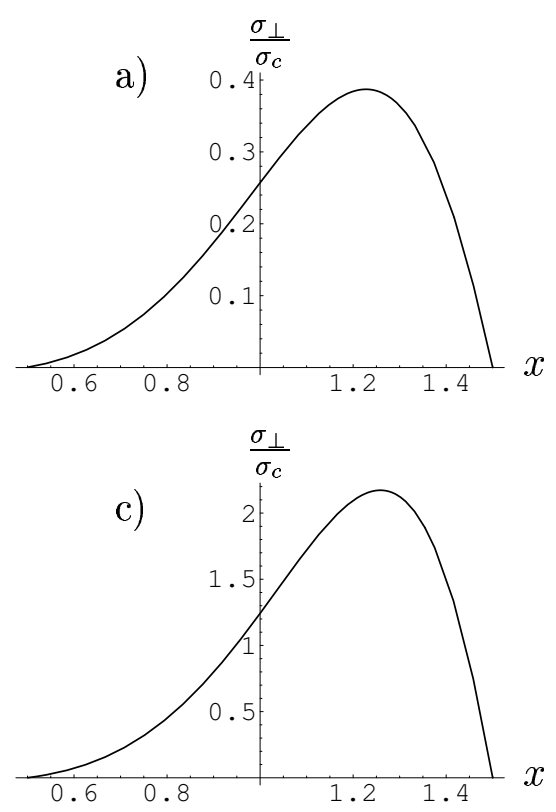
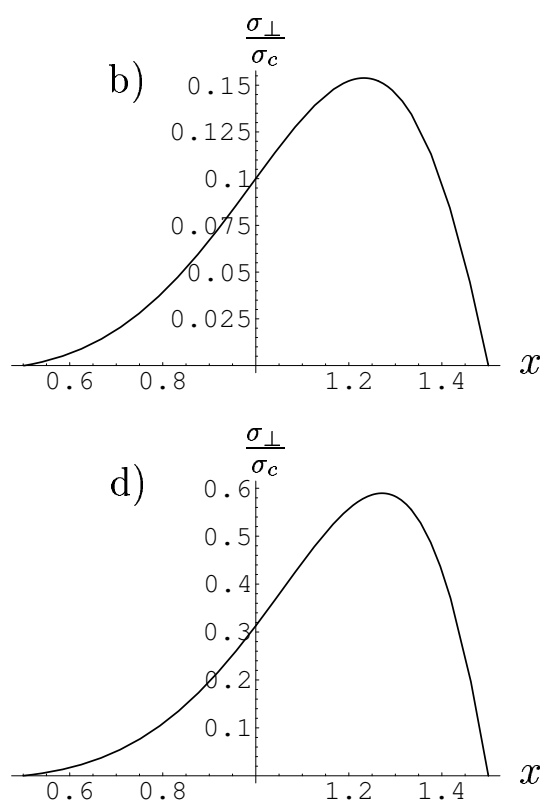

Figure 3: A set of curves demonstrating the oscillation of the profile of $\sigma_{\perp}$ on the midplane $z=0$, normalized with respect to a constant value $\sigma_{c}$, for "compressible" flow when the value of the function $\psi$ on the magnetic axis is flow dependent and the Mach number $M_{0}$ increases: a) $M_{0}=0.1$, b) $M_{0}=0.2$, c) $M_{0}=0.5$ and d) $M_{0}=0.6$. The aspect ratio is $\alpha=2 ; x \equiv R / R_{0}$ with the vertical axis being placed at the position of the geometric center $(x=1)$.
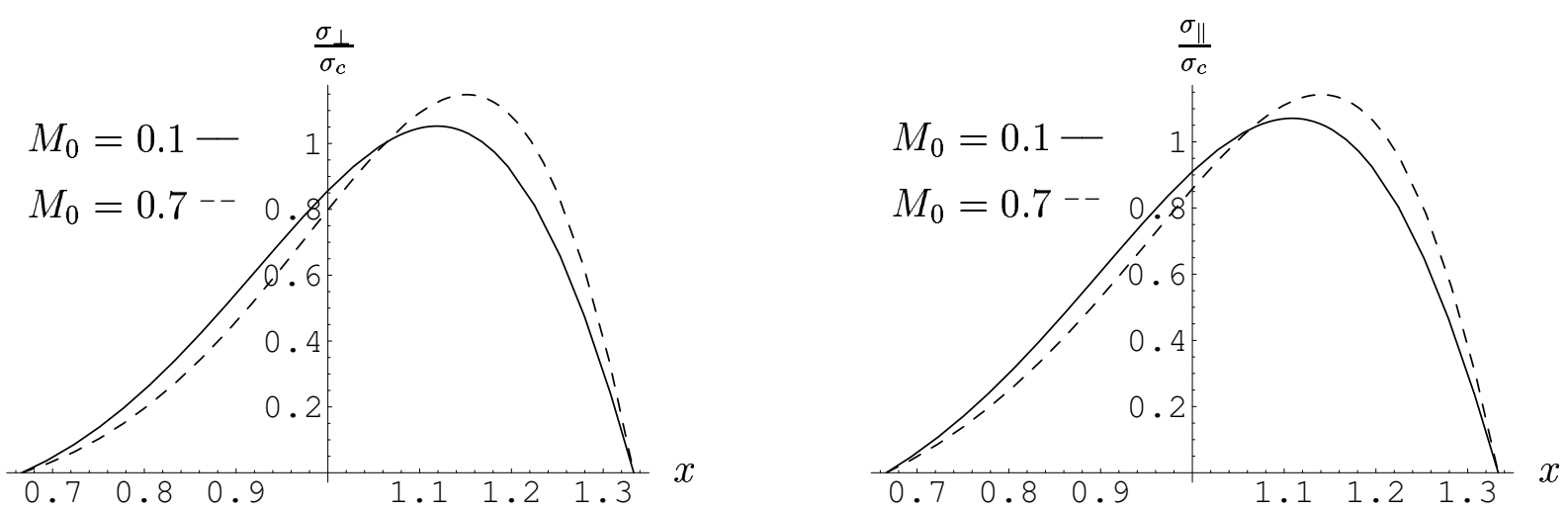

Figure 4: The figure shows the increase of the maximum of $\sigma_{\perp}$ and $\sigma_{\|}$and the displacement of its position outwards for "compressible" flow due to the increase of the Mach-number $M_{0}$. 

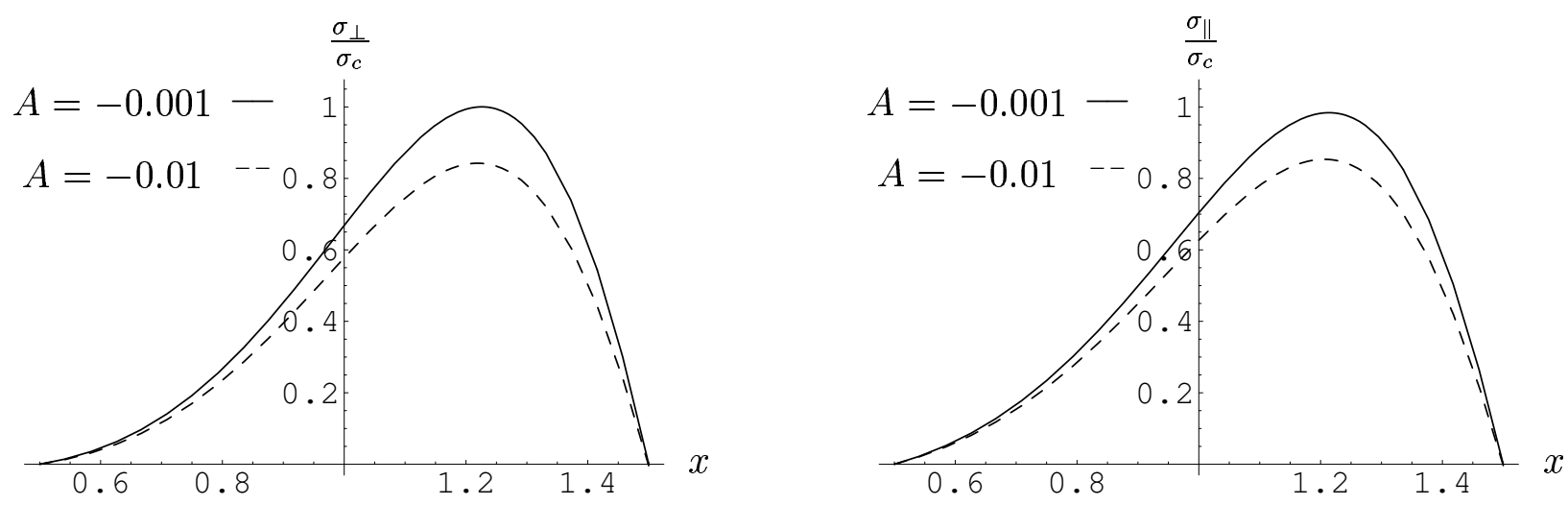

Figure 5: The figure shows the decrease of the maximum of $\sigma_{\perp}$ and $\sigma_{\|}$but the insensitivity of its position for incompressible flow as the parameter $A$ decreases.

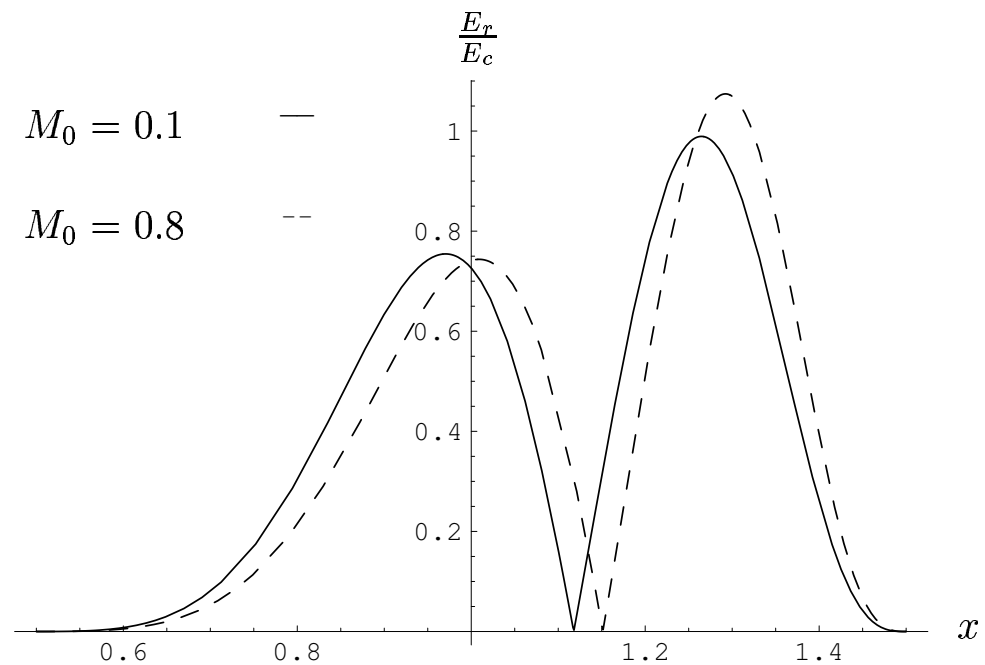

Figure 6: Electric field profiles on the mid-plane $z=0$ for "compressible" flow Comparison of the two curves indicates the increase of the local maxima of $\mathbf{E}_{p}$ and the outward shift of the their positions as the Mach number $M_{0}$ increases from 0.8 to 1 . The point in between the two maxima at which $\mathbf{E}_{p}=0$ corresponds to the magnetic axis. The aspect ratio is $\alpha=2$ and the value of the shaping parameter $n$ [Eq. (38)] is 3 . 


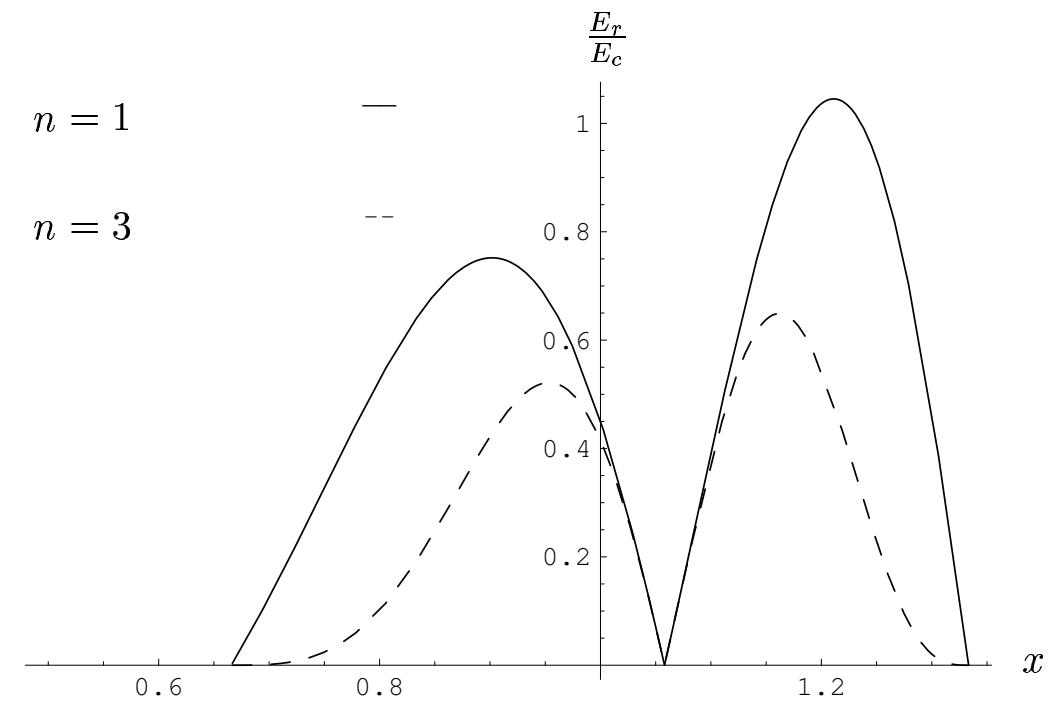

Figure 7: Electric field profiles on the mid-plane $z=0$ for $\alpha=3, M_{0}=0.4$ and two different values of the shaping parameter $n: n=1$ and $n=3$.

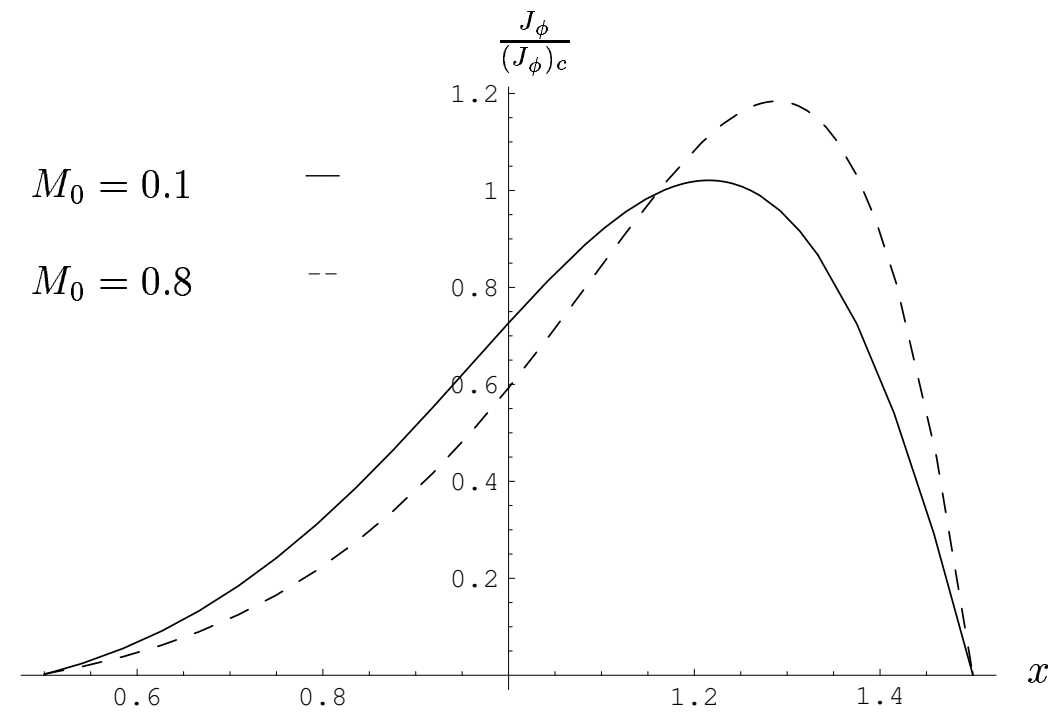

Figure 8: Toroidal current density profiles on the mid-plane $z=0$ for "compressible" flows Comparison of the two curves indicates the increase of the maximum and the outward displacement of its position as the Mach number $M_{0}$ increases from 0.1 to 0.8 . The aspect ratio is $\alpha=2$. 